The Sustainable City XII 125

\title{
URBAN REGENERATION WITH CARBON ECONOMY
}

\author{
BÜLENT YALAZI, TUĞÇE SÖNMEZ SANER, AYŞE ŞEYMA MANAP, \\ EVREN SAYIN \& ONAT ÇAĞATAY MUŞTA \\ Ministry of Environment and Urbanization, Turkey
}

\begin{abstract}
In Turkey, approximately 6.5 million dwelling units are deemed to be under disaster risks and it has become a government policy to rebuild this building stock. In order to fulfill the financial dimension of this urban regeneration attempt, the Ministry of Environment and Urbanization has developed a planning standard called "Sustainably Performed Urban Regeneration" SuPerUrban to mobilize the economies of sustainable living styles. Compared with the classical urban planning approaches, the environment friendly planning and urban design standard has focused on reducing the carbon footprint of the redevelopment and to create a new urban lifestyle in terms of the use of land, energy, water, natural resources and transportation. SuPerUrban reduces living costs of the households and local government expenditures and provide efficiencies that can be measured with the reduced percentage of the overall carbon footprint. Eventually, while being urban friendly it provides the means for the households to finance their share of the cost of regeneration with their reduced daily expenditures. In order to facilitate the model, carbon footprint calculation tool is developed to assist the project owners at both the neighborhood and building levels. Certain technology alternatives are provided to choose and compare the savings and reductions easily from the web. A pilot project is also developed using the standard and the tool in Eskişehir, Turkey. The technologies to be used in the project are intended to be produced in the area in order to provide jobs and create a green economy.

Keywords: Sustainability, Urban Regeneration, Ecological Labelling System, SuPerUrban.
\end{abstract}

\section{INTRODUCTION}

In the early part of the $21^{\text {st }}$ century, disasters threatening future of humanity, climate change and dependence to hydrocarbon are regarded as topics that a solution method has to be developed immediately on the international agenda. These topics which are raised as symptoms of dependence to non-renewable energy sources and to lifestyle accordingly these sources should not be approached separately and must be studied together. Precautions taken against to threats to our planet are going to show how our planet will be shaped and at the same time these precautions have a role in providing economic development trends.

Associated with Climate Change Adaptation, developing resistance to natural disaster, reducing heat island effect, carbon emission, conserving biodiversity, supporting organic and local agriculture, promoting sustainable energy source and energy efficiency targets are important topics of today's planning agenda. Therefore, these targets must be addressed in current planning studies. To reach these targets and to adapt these targets to our socio-cultural life, local and unique solutions should be developed.

Today, humanity understand that economic development due to carbon emission is not sustainable and begin to develop a new economic model in which development-oriented activities can be realized with the lowest possible carbon emissions. With this trend, the concept of "Low Carbon Economy" has emerged as the economic development model of 21 st century. This concept gives a different perspective to climate change/disasters and dependence to non-renewable energy sources. It is possible to generate an economic model which causes minimum greenhouse gases emission when a value generated by the Low Carbon Economy, which plays a role in solving problems in both topics, is operationalized.

Another important role of Low Carbon Emission is to provide a chance to realize world's new trend with urban regeneration operations. Urban regeneration which is carried out by 
sustainability principles is emerged as an important tool for our country to meet its national obligations on climate change. In order to use this tool more efficient and to transform the areas under disaster risk into more healthy and durable places, The Law of Transformation of Areas under the Disaster Risks (Law No. 6306) has been put into force in 2012.

The Law of Transformation of Areas under the Disaster Risks has been put into force in order to reduce possible damages of earthquake and other natural disasters. However, if urban regeneration is implemented by taking measures to reduce carbon emissions, households can provide savings from energy, water and transportation expenses. These savings can be used for repayment of urban regeneration credits. In the framework of this idea, first steps of an "Ecological Settlement Unit" have been taken by Directorate General of Infrastructure and Urban Transformation Services.

By the year 2015, the country has reached the resource limits of the existing policy observed in urban transformation and has entered a trend of deceleration and the rent subsidy assistance provided for urban transformation has decreased. Therefore, the method used to finance the urban transformation by the carbon economy savings has begun to work on a project to open a new page in the urban transformation sector. In other words, Low Carbon Economy will play a key role to realize urban transformation.

By using savings generated by Low Carbon Economy and lifestyle developed independently from hydrocarbons on financing of urban transformation, an environmentally friend cities, production and consumption could be achieved. This achievement will create sustainable, localized, energy efficient and resistant society that uses its own resources. In order to reach these targets, Ministry of Environment and Urbanization started to carry a pilot project called "Sustainably Performed Urban Regeneration" SuPerUrban in Kocakir Province, Eskişehir to obtain an ecological settlement unit standard.

In this context, in the Law no. 6306 frame, "neighborhood standards" have been developed that aim to obtain SuPerUrban neighborhoods with ecological characteristics. Within the scope of this standard, social, economic and environmental criterion for the implementation of urban transformation has been determined. Especially, it is aimed that the criticism on the question of the social effects of transformation practices not being measured will be abandoned by this urban transformation model based on the socioeconomic sustainability principles laid out in the standard.

With "Sustainably Performed Urban Regeneration" SuPerUrban, a new concept of urban transformation and a new model has been defined and the implementation of this model is planned to be carried out through the project named as "Kocakır Ecologic Settlement Unit Project" which can be considered as a small satellite city that can satisfy its need by its own sources.

The implementation of this new model is planned to be carried out in the Reserve Building Area of 838 hectares in Kocakır District of Eskişehir. The project will be carried out in five stages and around 45.000 people will live in project area. When the project is completed the value of Eskişehir will increase and the welfare level of the city will increase.

\section{2 "SUSTAINABLY PERFORMED URBAN REGENERATION" SUPERURBAN AND "KOCAKIR ECOLOGIC SETTLEMENT UNIT"}

"Kocakır Ecologic Settlement Unit Project" is a first in terms of setting a standard that will provide carbon reduction for residential areas both in our country and in the world through its labeling system.

The application of this new model, which will open a new page in urban transformation, is located $11 \mathrm{~km}$ from Eskişehir City Center, also it is in the center of Istanbul and Ankara and these cities can be reached by high-speed train. The main reason why this project is 


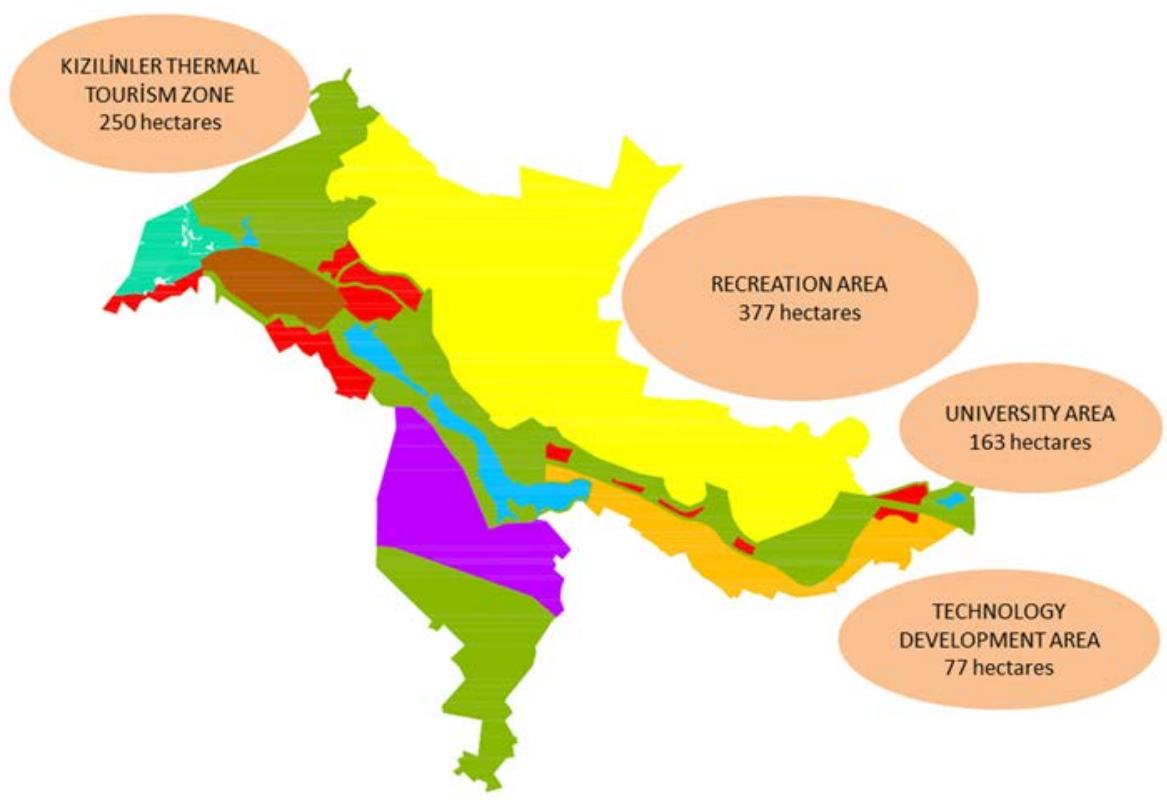

Figure 1: Eskişehir Metropolitan area central region 1/25.000 scale master plan.

implemented on Kocakır District is presence of the reserve building area of 838 hectares which will provide suitable space for the project to be carried out in the area. Absence of agricultural land, endemic and protected species, forest, risk of disaster in the area are the other good characteristics of Kocakır District. Moreover, there is solar and wind renewable energy potential in the region.

The project also contributes to the macro form of Eskişehir city. The project area is associated with the Kizilinler thermal tourism zone, the third university area and the olympic village recreation area, which are located nearby with applications done on the 1/25.000 scale master planning and plan decisions [1]. The welfare of the project area will increase and the value of the area will make the area a center of attraction with its nearby.

The project, aiming to develop a standard for residential areas alongside buildings, sets out general standards for these new settlements, which minimize harmful environmental impacts of buildings and settlements and enhance compliance with the ecosystem. Minimum criteria and performance criteria have been defined in six main criteria (land use and urban design, water, energy, transportation, materials and sources and socio-economic sustainability) within the framework of these standards. All these criteria will be called "Sustainably Performed Urban Regeneration" SuPerUrban [2].

\subsection{Innovations of SuPerUrban system according to other systems}

All world examples related to "Sustainably Performed Urban Regeneration" SuPerUrban such as BREEAM Community (England), DGNB District (Germany), HQE Quartier (France) and LEED Neighborhood (USA) had been examined before the protocol for 
SuPerUrban was prepared. It is considered that he most comprehensive system among these systems is the LEED Neighborhood Certification System.

In this manner, it is stated in the protocol to prepare the project will be based on the LEED Neighborhood Certification System [3]. In addition to this, the project will be developed to include all the other leading certification systems in the world and to be over them.

Project has introduced the concept of climate change adaptation criteria, neighborhood modelling in energy, waste water treatment criteria, neighborhood life cycle analysis and minimizing transportation demand in field and surroundings in the existing system in Turkey. In addition, a socio-economic sustainability criterion, which is not available on the other systems, is another innovative approach that the project has introduced.

SuPerUrban Label System, which is based on many progressive system and making them more comprehensive, aims to provide sustainability in structures and neighborhoods in accordance with existing building legislation [4].

The SuPerUrban label system has been developed as a certification system which is over all other certification systems and has been made more comprehensive and superior to all other systems by changing the existing evaluation system in accordance with the needs of Turkey.

\subsection{SuPerUrban label system - evaluation system}

A comprehensive rating and labeling system has been developed as the first step in the realization of the ecological settlement unit in order to measure the benefit that will be achieved as a result of the specified standards, and the minimum criteria and performance criteria have been set in this direction.

The methodology adopted in the SuPerUrban Label System is a system operation that scales ecological settlement units as how the world counterparts do and at the same time internalizes national norms and is flexible enough to integrate with the world system in the future. SuPerUrban Label System not only focus on environmental impacts, but also allow to reach the achievement of a certain level of economic and social impacts. In respect to this, such effects, which are more difficult to quantify with an EPD system, is thought to be associated with a labelling system. So, in addition to EPD System, ISO 14024 Type-I ecolabel structure is deemed to be created. With the label system to be used by the Ministry of Environment and Urbanization, settlements can be labelled and different incentives and financial models would be applied to the ecological settlements units.

In order to get SuPerUrban Label, an ecological settlement unit must be labelled in six main criteria: Land Use and Urban Design, Energy, Water, Transportation, Materials and Sources, Socio-economic Sustainability [5].

\subsubsection{Land use and urban design}

In this criterion title, the rules that are required in an ecological settlement unit are defined in planning, urban design and application phases. To select environmentally sensitive land for a sustainable planning approach, to increase the carbon sink potential of the area after urban planning compared to pre-planning, to form mix-use neighborhoods with archaic urban fabric and to present different housing typologies to live different income groups together have been identified as key objectives. An approach to urban design was adopted in which residential and business places are within walking distance with walkable streets. 


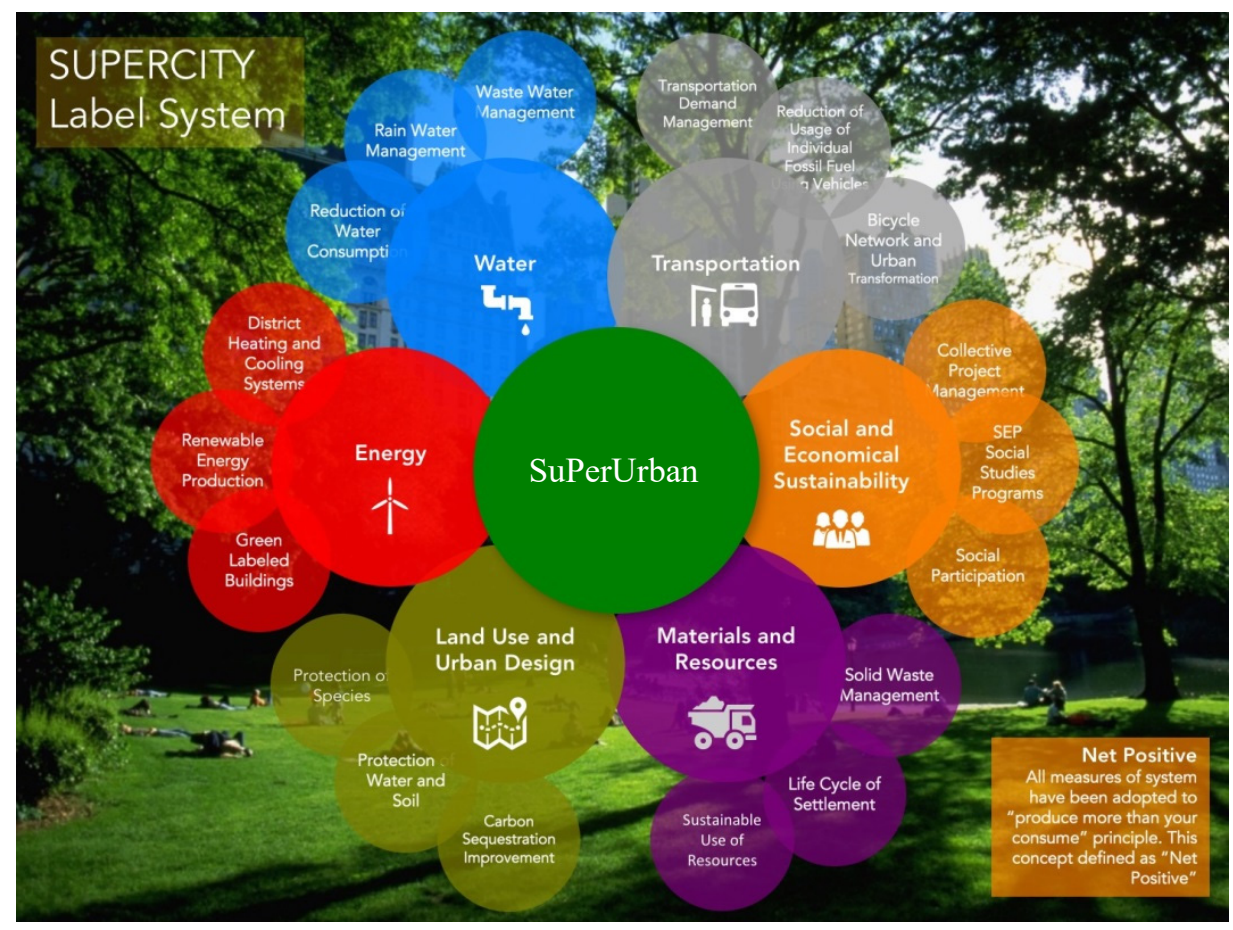

Figure 2: SuPerUrban system schematics.

\subsubsection{Energy}

In this criterion title, to create an ecological settlement with green buildings that is energy efficient, solar oriented and cost optimal, to reduce the heat island effect, to use central heating and cooling systems in district scale have been identified as key objectives. In addition to these, the principle of generating the energy demand of the ecological settlement in project area is adopted.

\subsubsection{Water}

In this criterion title, to reduce regional, indoor and outdoor water consumption, to establish rainwater and wastewater management systems and to produce ecological settlements own water needs have been identified as key objectives.

\subsubsection{Transportation}

It is aimed to reduce transportation-related energy consumption and emissions by introducing a holistic approach to urban transportation within this criterion. For this reason, strategies that intra-city transportation requests to be directed to walking, cycling and using lowemission public transport vehicles have been adopted. It has been aimed to meet the transportation energy need from the renewable sources. Also, high quality transportation facilities have been created by creating compact residential units connected to the environment that reduces travelling distances between residential and commercial. 


\subsubsection{Materials and sources}

This criterion encourages the use of construction materials that have a low environmental impact in infrastructures and buildings and construction materials manufacturers to produce these materials.

\subsubsection{Socio-Economic Sustainability}

In this criterion, in the ecological settlements, it is essential for the project to create value added community and an environmentally conscious society. It is aimed that the people to be active as much as the investors are in the urban transformation operation and to take an active organized role in all the processes.

These six main strategies aim to determine the main standards for planning an ecological settlement while evaluating this ecological settlement in terms of sustainability criteria. Carbon footprint performance is taken into account when evaluating settlements and determining public support with these obligatory criteria. The carbon emission effect of some criteria, the carbon sink effect of some criteria, and the measurable performance of other criteria is taken into account to calculate performance of ecological settlement.

The principle of producing more than consuming in the SuPerUrban System has been adopted and this is called the "Net Positive" concept. All greenhouse gas emissions are accounted for and calculated on the basis of carbon emissions in this system. With this feature, SuPerUrban is an environmental impact assessment tool that makes a scientific measurement of the settlement units according to the global warming potential of settlement. In order to reduce carbon footprint of a settlement throughout whole lifecycle, a "Carbon Calculator Tool" has been obtained to make a lifecycle analysis to estimate how much carbon will be emitted [6]. Within this scope, settlement units are rated with six performance levels which are Base, Base,$+ \mathrm{B}+, \mathrm{A}+, \mathrm{Net} \sim$ and $\mathrm{Net}+$. These performance levels are shown in Table 1.

\subsubsection{Methodology}

Data entries based on these six main criteria are registered in the Carbon Calculator Tool. The carbon calculator analyzes the input data according to $\mathrm{CO}_{2}$ emission values and gives information about the settlement place. The amounts of interest support to be given to the area of urban regeneration are determined according to the results.

Methodology is the calculation of the life cycle effect of a settlement site based on $\mathrm{CO}_{2}$. In addition to these calculations, the life cycle cost of the settlements and the household savings are compared to each other to determine social and economic sustainability. As a result of these detailed calculations, interest support to be applied in the urban transformation

Table 1: SuPerUrban performance levels based on reduction of carbon emission.

\begin{tabular}{|c|c|}
\hline Level & $\begin{array}{c}\text { Reduction of } \mathrm{CO}_{2} \\
\text { Emission }\end{array}$ \\
\hline Net + & $>100 \%$ \\
\hline Net $\sim$ & $>80 \%$ \\
\hline A + & $>60 \%$ \\
\hline B + & $>20 \%$ \\
\hline Base + & $>0 \%$ \\
\hline Base & $=0 \%$ \\
\hline
\end{tabular}


area will be determined by Ministry of Environment and Urbanization. TS EN 15978 the method of building life cycle analysis has been adopted in the settlement site Life Cycle Analysis approach.

The GHG Protocol, published by the World Resources Institute (WRI) and the World Sustainable Development Council (WBCSD) and in addition to this IPCC-based methodology PAS2070 is based on calculating the amount of $\mathrm{CO}_{2}$ emissions [7].

For the accuracy of the system, all carbon emission sources in the settlement area will be determined and the effect will be considered throughout the year.

\subsection{SuPerUrban system structure and organizational model}

SuPerUrban Label System is not a new system alternative to Turkey's existing planning system. But this system is a new approach that introduces innovative practices that will reduce negative environmental impacts within existing legislation. This system has been a first to handle ecological settlement and urban regeneration together.

The SuPerUrban Label System has unique training system and expertise documents. Starting with building material production, green building construction, ecological settlement planning and construction processes will be evaluated along with integrated design understanding and participation processes. Projects will be valued by integrated expert assessment teams and incentives will be given according to project performance.

In the SuPerUrban System, the design of ecological settlements and green buildings will be carried out by integrated planning and design teams consisting of interdisciplinary experts [8]. In this direction, there are Integrated Planning and Designing Teams and Integrated Expert Inspection and Approval Teams within the system. Training programs for label system experts will be provided by expert training teams authorized by the Ministry of Environment and Urbanization. Training contents will be created by expert training teams also authorized by the Ministry. Experts graduated from these training programs will be certified in the areas of specialization they are trained in.

The SuPerUrban Labeling System consists of 29 minimum and 28 performance criteria in 6 main criteria. Therefore, each criterion requires a separate approval process, as too much technical requirement is required. After the urban planning process of the integrated design team, Integrated Expert Inspection and Approval Teams, which has been trained for each criterion, inspect and approve the plans.

Social participation is one of the important elements of the SuPerUrban Label System. The participation of the householders should continue not only in the design process but also in the construction and lifecycle of the settlement. This new organizational structure aims to ensure that ecological settlement performance level does not change during the life cycle of the settlement and that their performance continues at the same level. This new organization is called "Community Management" [9]. Community Management can improve the performance level of the settlement according to the developing technologies in time.

SuPerUrban Labeling System evaluates the settlements in three stages that are Conceptual project, preliminary project and application project.

The conceptual project is the stage in which the goals of the project are set out before the project starts. Counterparts can argue and make a decision about the project in this stage. This process represents the decision period before the application to the Ministry.

The preliminary project is the stage in which quantities are determined, the sizes are revealed and the spatial characteristics of settlement are planned. It is necessary to come to a mutual agreement with the householder after this stage. 
The application project is the stage in which all the decisions related to the project are taken and the final shape of the project is obtained. the most precise measurement of the project is carried out at this stage.

All works done for "Kocakır Ecologic Settlement Unit Project" are preliminary projects. The application projects are being prepared for the realization of this project.

\section{CONCLUSIONS AND EVALUATION}

When evaluating the six key strategies developed within the "Kocakır Ecologic Settlement Unit Project", it is seen that the project fulfills all targets set during the planning phase and achieves the emission reduction in the direction of performance in these criteria.

When the performance level of the targets determined in the six key strategies, "Kocakir Ecologic Settlement Unit Project" succeed these scores:

On the Land Use and Urban Design Strategies, the project has increased it carbon sink area by $140 \%$ and reaches "Net + " level. To achieve this result, low density settlement design, small lot coverage and increased plant density were used. Floor area ratio is 0.37 for whole project area as it is 1.4 for residential area and the lot coverage is 0.08 for the project. It is estimated that monthly income of $162 \mathrm{TL}$ per household will be obtained with the savings provided by Land Use and Urban Design.

On the Energy basis, carbon emissions have been reduced by $83.24 \%$ and reached Net $\sim$ level. In order to reach this level, cogeneration system, heat pumps, micro grids and PV solar panels installation is planned. Also, buildings are directed to the sun and heat island effect is set to be zero. In this way, the infrastructure has been made to be $75 \%$ efficient and monthly income of 385 TL per household will be obtained.

Water saving was calculated as $91.3 \%$ and Net level was reached. In order to get this level, water consumption reduced by $75 \%$ for irrigation and all water demand is met from gray water systems and rain water collection system. Householders can save up to 72 TL from water bills.

Transportation-related carbon emissions decreased by $92 \%$ that means Net $\sim$ level has been reached. $26 \%$ walking, $13 \%$ cycling, $55 \%$ public transport and $6 \%$ private vehicle use is aimed to catch this level. In the project area, it is planned that EV, PHEV, biodiesel and CNG based vehicles to be used. Householders can save up to 196 TL from transportation costs.

Carbon emissions in material and resources criteria were calculated as $65 \%$ and $\mathrm{A}+$ level has been reached. In order to achieve this level, it is aimed to provide $25 \%$ recycled content for infrastructure, $50 \%$ recycled content for buildings and $97 \%$ of debris recycling.

Projects done to provide the social and economic sustainability, urban agriculture, employment opportunities provide $115.6 \%$ added value for householders and Net+ level has been reached.

When all the criteria are considered together throughout the project, 97.93\% carbon emission reduction, which is over the European standards, has been achieved and Net $\sim$ level has been reached. In the other words, householders can save up to 815 TL from living expenses [10]. In addition, Banks can provide zero-interest loans to householders through government support and the low interest foreign-funded loans for ecological settlements unit. So, this amount corresponds to $87 \%$ of the households' urban regeneration credits repayments. Thus, a significant contribution is provided to home and country economies.

When people find the opportunity to live in a healthier and safer place, the energy dependence to foreign countries of Turkey will be reduced by "Kocakır Ecologic Settlement Unit Project". Considering increased house value, householder has a chance to own an upperclass residence by paying low rent. 
By the SuPerUrban Label System, people will be given to an opportunity to live in sustainable places and sample cities. Also, technologies developed within the project will be a new source of innovation for the construction sector of Turkey. The draft "Regulation on Sustainably Performed Urban Regeneration" was prepared and submitted for receiving opinions of the relevant institutions and organizations. The Regulation is ready to be sent to Prime Ministry to introduce law.

\section{REFERENCES}

[1] Eskişehir Metropolitan Municipality. Eskişehir Metropolitan Area Central Region 1/25.000 Scale Master Plan Analysis Report, 2014.

[2] Ministry of Environment and Urbanisation. Kocakır Reserve Building Area Ecological Settlement Unit Standard Development Project Analysis-Synthesis and Component Reports, Chapter 1-1, 2015.

[3] http://www.usgbc.org/leed

[4] Ministry of Environment and Urbanisation. Kocakır Reserve Building Area Ecological Settlement Unit Standard Development Project Analysis-Synthesis and Component Reports, Chapter 1-2, 2015.

[5] Ministry of Environment and Urbanisation. Kocakır Reserve Building Area Ecological Settlement Unit Standard Development Project Analysis-Synthesis and Component Reports, Chapter 1-1, 2015.

[6] Ministry of Environment and Urbanisation. Kocakır Reserve Building Area Ecological Settlement Unit Standard Development Project Analysis-Synthesis and Component Reports, Chapter 1-8, 2015.

[7] Ministry of Environment and Urbanisation. Kocakır Reserve Building Area Ecological Settlement Unit Standard Development Project Analysis-Synthesis and Component Reports, Chapter 1-8, 2015.

[8] Ministry of Environment and Urbanisation. Kocakır Reserve Building Area Ecological Settlement Unit Standard Development Project Analysis-Synthesis and Component Reports, Chapter 1-12, 2015.

[9] Ministry of Environment and Urbanisation. Kocakır Reserve Building Area Ecological Settlement Unit Standard Development Project Analysis-Synthesis and Component Reports, Chapter 1-18, 2015.

[10] Ministry of Environment and Urbanisation. Kocakır Reserve Building Area Ecological Settlement Unit Standard Development Project Analysis-Synthesis and Component Reports, Chapter 4-6, 2015. 